\title{
Definition of Plant-Pathogenic Pseudomonas Genomospecies of the Pseudomonas syringae Complex Through Multiple Comparative Approaches
}

\author{
Simone Marcelletti and Marco Scortichini
}

\begin{abstract}
First and second authors: Consiglio per la Ricerca e la Sperimentazione in Agricoltura (C.R.A.); first author: Centro di ricerca per la Frutticoltura, Via di Fioranello, 52; I-00134 Roma, Italy; and second author: Unità di ricerca per la Frutticoltura; Via Torrino, 3; I-81100 Caserta, Italy.
\end{abstract}

Accepted for publication 20 May 2014.

\begin{abstract}
Marcelletti, S., and Scortichini, M. 2014. Definition of plant-pathogenic Pseudomonas genomospecies of the Pseudomonas syringae complex through multiple comparative approaches. Phytopathology 104:1274-1282.

A total of 34 phytopathogenic strain genomes belonging to the Pseudomonas syringae species complex and related species, including many pathotype strains, were assessed using average nucleotide identity (ANI) analysis. Their taxonomic relationships were consistently confirmed by the tetranucleotide frequency correlation coefficient (TETRA) values, multilocus sequence typing analysis (MLSA) performed with seven housekeeping genes, using both maximum likelihood and Bayesian methods, and split consensus network analyses. The ANI, MLSA, and split consensus analyses provided consistent and identical results. We confirmed the occurrence of the well-demarcated genomospecies inferred

our analyses. Genomospecies 1, 2, 4, 6, and 9 resulted well demarcated, whereas strains of genomospecies 3 and 8 had ANI values between 95 and $96 \%$ in some cases, confirming that this threshold reveals very closely related species that might represent cases of splitting entities or the convergence of different species to the same ecological niche. This study confirms the robustness of the combination of genomic and phylogenetic approaches in revealing taxonomic relationships among closely related bacterial strains and provides the basis for a further reliable demarcation of the phytopathogenic Pseudomonas species. Within each species, the pathovars might represent distinct ecological units. The possibility of performing extensive and standardized host range and phenotypic tests with many strains of different pathovars can assist phytobacteriologists for better determining the boundaries of these ecological units.
\end{abstract} sensu Gardan et al. using DNA-DNA hybridization and ribotyping analyses. However, some $P$. syringae strains of the pathovars morsprunorum and lachrymans were placed in different genomospecies in
Additional keywords: genome comparison, polyphasic taxonomy.
The genus Pseudomonas includes metabolically versatile species capable of living as parasites and/or saprophytes in the most diverse terrestrial habitats, including plants (38). Some Pseudomonas spp. damage certain cultivated and wild plants and mushrooms. Twenty-one plant-pathogenic Pseudomonas spp. have been described $(5,6,22)$. Among these, $P$. syringae shows a composite ecology of species populations. In fact, this species currently encompasses 57 different pathovars (5), known as the "P. syringae complex," capable of causing disease to herbaceous and woody crops belonging to both mono- and dicotyledonous plant species. A complex is defined as a single species composed of distinct populations capable of infecting a limited number of host plants $(13,53)$. Such distinct populations can show limited or incomplete reproductive isolation resulting in poorly defined species boundaries. The pathovar (i.e., pathogenic variety) concept was introduced in the 1980s to clarify phytosanitary legislation. This concept enables the identification and naming of diverse plant diseases of bacterial origin to aid the development of appropriate detection techniques and/or quarantine protocols. Pathovars are defined as "strains or set of strains with the same or similar characteristics, differentiated at intrasubspecific level from

Corresponding author: M. Scortichini;

E-mail address: marco.scortichini@entecra.it

* The $e$-Xtra logo stands for "electronic extra" and indicates that the online version contains two supplemental tables.

http://dx.doi.org/10.1094/PHYTO-12-13-0344-R

(C) 2014 The American Phytopathological Society other strains of the same species or subspecies on the basis of distinctive pathogenicity to one or more plant hosts" (14). Recent studies suggest that pathovar-host specificity is mainly due to the presence of typical repertoires of the type III secretion system effector proteins, which allow the pathovar to overcome host plant defense mechanisms (33).

Although the pathovar has no formal standing within the International Code of Nomenclature of Bacteria (29), which provides rules up to the subspecies level, it is recognized as an effective biological entity (15) and its existence is regulated under the auspices of the International Society of Plant Pathology. In addition, this specific term was initially considered to be a temporary categorization (14) because at that time few plant-pathogenic bacterial species could be differentiated by phenotypic tests as required by the Approved List of Bacterial Names (50). A comprehensive attempt to clarify the taxonomic relationships within the pathovars of the $P$. syringae complex was performed by Gardan et al. (16) using DNA-DNA hybridization (DDH) and ribotyping analyses. The authors assessed 48 pathovars of the complex and eight related species and proposed nine discrete genomospecies. Each genomospecies comprises strains, including the type-strain, exhibiting at least $70 \%$ DNA-DNA homology. However, according to the Bacteriological Code, a genomospecies should be named formally only when differentiating phenotypic characteristics are available (58). As Gardan et al. (16) could not provide reliable phenotypic traits (e.g., carbon source assimilation) to clearly distinguish all nine genomospecies, they could officially describe only two species ( $P$. cannabina and $P$. tremae). Consequently, most genomospecies still need to be formally described. 
The definition of a bacterial species is difficult. Currently, DDH, 16S rRNA gene sequence analysis, and multilocus sequence typing analysis (MLSA) are the techniques commonly utilized to genetically determine the bacterial species, with MLSA providing a good combination of reliability and repeatability (1) (see also Plant Associated and Environmental Microbes Database; www. pambd.org/). As far as phytopathogenic pseudomonads are concerned, MLSA studies provided fundamental insights into their phylogenetic and taxonomic relationships $(4,8,9,36,43)$.

However, each of these techniques has some limitations, such as the impossibility of assembling cumulative databases based on DDH and its reliability only for closely related species or subspecies, the low variability and conservative nature of 16S rRNA genes not permitting sufficient resolution to infer clear taxonomic relationships, and the occurrence of multiple $16 \mathrm{~S}$ rRNA genes and the putative bias in the selection of the housekeeping genes for MLSA (41,42). Recently, the average nucleotide identity (ANI) analysis of conserved and shared genes between two bacterial strains based on pairwise genome comparisons has been proposed as a new standard for the definition of prokaryotic species (27). The bacterial genome assessment inferred using ANI well represented the degree of evolutionary distance between the compared genomes, and an ANI value of $94 \%$ was proposed to replace the classic DDH value of $70 \%$ for species demarcation (27). A more extensive study confirmed the reliability of such an analysis and noted a slightly narrower boundary of $95 \%$ identity for the consistent substitution of the DDH value of $70 \%$ (20). In confirming the robustness of the ANI analysis, it established the species demarcation boundary at a value of 95 to $96 \%$ identity and suggested further confirmation by the assessment of the tetranucleotide frequency correlation coefficient (TETRA) value (41). However, the combination of MLSA and genomic analysis, providing either a phylogenetic view or a pairwise genome comparison of all shared orthologous protein coding genes, could represent a robust approach for the reliable demarcation of bacterial species $(11,17,42,45,51,52,54)$.

In this study using ANI and TETRA analyses, MLSA, both ML and Bayesian methods, and a consensus split network with seven housekeeping genes, we examined the currently available genomes of 34 plant-pathogenic strains of $P$. syringae pathovars and some related species representing seven of the nine genomospecies identified by Gardan et al. (16). Two genomospecies, genomospecies 5 ( $P$. tremae) and 7 ( $P$. syringae pv. tagetis and $P$. syringae pv. helianthi), could not be assessed because the genomes of these pathogens are not deposited in the databanks. The genomes of $P$. fluorescens A506 and P. putida UW4 were used as outgroups. We confirmed the existence of well-demarcated genomospecies within the $P$. syringae species complex in agreement with the DDH and ribotyping study of Gardan et al. (16). In addition, by revealing consistent ANI values between 95 and $96 \%$, we highlighted the close relationship between genomospecies 3 and 8 . The present analysis includes many pathotype strains of the $P$. syringae pathovar complex and thus provides the basis for a further reliable demarcation of plant-pathogenic Pseudomonas species through ANI/TETRA and MLSA analyses.

\section{MATERIALS AND METHODS}

Library preparation, genome sequencing, and assembly. For genomic sequencing, the DNA of $P$. syringae pv. coryli NCPPB 4273, pathotype-strain of the pathovar, was prepared as described elsewhere $(36,46)$. Briefly, bacterial genomic DNA was extracted from $1 \mathrm{ml}$ of overnight cultures grown in $\mathrm{KB}$ broth using a Wizard DNA purification kit (Promega Italia, Padova, Italy) following the manufacturer's instructions. DNA was measured and checked for quality using a NanoDrop (NanoDrop Products, Wilmington, DE). A total of $10 \mu \mathrm{g}$ of DNA from each sample was fragmented by incubation for $70 \mathrm{~min}$ with $5 \mu \mathrm{l}$ of dsDNA
Fragmentase (New England Biolabs, MA). The reaction was stopped with EDTA and purified using a QIAquick PCR purification kit (QIAGEN, Hilden, Germany). The eluate was end repaired using an End Repair kit (New England Biolabs) for $30 \mathrm{~min}$ at $20^{\circ} \mathrm{C}$. The end-repaired DNA was A-tailed for $30 \mathrm{~min}$ at $37^{\circ} \mathrm{C}$ using a d-A Tailing kit (New England Biolabs). After purification using the MinElute purification kit (QIAGEN), the DNA was ligated using Quick T4 DNA ligase (New England Biolabs) to $500 \mathrm{pmol}$ of Illumina adaptors that had been previously annealed by heating at $98^{\circ} \mathrm{C}$ for $3 \mathrm{~min}$ and then slowly cooling to $16^{\circ} \mathrm{C}$ in a thermocycler. After further purification using the MinElute purification kit (QIAGEN), $1 \mu \mathrm{l}$ of each reaction was quantified by labeling with biotin, spotted on nitrocellulose after a serial dilution, and detected using an anti-biotin-AP conjugate (Roche Diagnostics, Monza, Italy) following manufacturer's instructions. Equal amounts of DNA from samples were pooled together and size fractionated by $2 \%$ MS-6 agarose (Conda, Madrid, Spain) gel electrophoresis in TAE buffer at $120 \mathrm{~V}$ for 60 min. Genomic DNA was sequenced using Illumina Genome Analyzer IIx (Illumina, San Diego, CA) at the Istituto di Genomica Applicata (Udine, Italy). In order to remove contaminants and adaptors, quality check of reads was performed using the extended randomized numerical alignEr (ERNE) filters, a string alignment package to provide tools to handle short reads (www. erne.sourceforge.net). Paired reads of $100 \mathrm{nts}$ were assembled into contigs using the de novo (i.e., without using a reference genome) assembly option of the CLC genomic workbench (CLCbio, Aarhus, Denmark) by setting the default parameters. Contigs sequences were scanned for open reading frames by GLIMMER, version 3.02. (12) which had been previously trained on the complete genome sequences of $P$. syringae pv. tomato DC3000 (NC_004578.1, i.e., Pto DC3000), P. syringae pv. phaseolicola 1448A (NC_005773.3, i.e., Pph 1448A), and P. syringae pv. syringae B728a (NC_007005.1, i.e., Psy B728a). The putative proteins were annotated against the RefSeq database using a PERL script for recursive BLASTX searches (2) and MUMMER software (28).

Public sequence data. In addition to the newly sequenced $P$. syringae pv. coryli strain, the genome data from 35 Pseudomonas strains were downloaded from the National Center of Biotechnology Information (NCBI) and from DOE Joint Genome Institute/Integrated Microbial Genome (JGI/IMG-ER) GenBanks. These data included genome sequences of $31 P$. syringae pathovars, and $P$. avellanae, $P$. viridiflava, and $P$. cannabina $\mathrm{pv}$. alisalensis strains as well as of $P$. fluorescens A506 and $P$. putida UW4 as outgroups (Table 1).

ANI and TETRA analyses. To evaluate the taxonomic relationships of 34 strains of phytopathogenic $P$. syringae pathovars and related species, ANI and TETRA analyses were performed. The analyses of sequences for the determination of their relatedness according to ANI and TETRA were performed with the software JSpecies (41). The analysis regarded 34 genomes belonging to seven out of nine $P$. syringae genomospecies sensu Gardan et al. (16) (Table 1). Due to current absence of some genomes in database, strains of genomospecies 5 and 7 were not analyzed. In addition, $P$. fluorescens A506 and P. putida UW4 were included in the assessment as outgroups. ANI was calculated using the MUMmer algorithm implementation (i.e., ANIm) (25). TETRA was used as an alignment-free genomic similarity index as oligonucleotide frequencies carry a species-specific signal. The use of a tetranucleotide usage pattern has been shown to be a good compromise between signal strength and needed computational power (41). Pairwise comparison between genomes is performed by plotting the corresponding tetranucleotide frequency and then obtaining a regression line.

Taxonomy based on MLSA and consensus split networks. To further evaluate the taxonomic relationships of the 34 strains of $P$. syringae and related species, MLSA was also carried out. 
$P$. fluorescens A506 and $P$. putida UW4 strains were used as outgroups. The maximum likelihood (ML) and Bayesian analyses were performed with nucleotides using seven housekeeping genes (dnaQ, gltA, pheS, recA, rpoB, serS, and tyrS), for a total of 3,003 nts. This set of genes was used to obtain a longer gene sequence to analyze than the classical set (i.e., gap1, gltA, gyrB, and $r p o D$ ). ML analysis was inferred with PhyML version 3.0 (21), with 1,000 bootstrap replicates, whereas for the Bayesian method we used MrBayes version 3.2.1, with 1,000,000 generations (http://mrbayes.sourceforge.net). To select the best fit model for both ML and Bayesian analyses, we used a phymltest procedure implemented in the R package APE (40). JC69 was used as best substitution model for both analyses. The robustness between the ML gene trees was tested by applying the Shimoidara-Hasegawa (SH) test (48) using the CONSEL program (49). The trees were visualized using FigTree software, version 1.1.2 (http://tree.bio.ed.ac.uk/software/figtree/). In addition, a consensus split network tree for inferring unrooted phylogenetic networks, based on concatenation of 3,003 nucleotides from the 34 phytopathogenic pseudomonads and $P$. fluorescens A506 and P. putida UW4 strains as outgroups, was built using the neighborjoining (NJ) algorithm with the Hamming distance method, obtained using the Splits-Tree4 software (23). Bootstrap analysis with 1,000 replications was performed by using the same software.

\section{RESULTS}

Genome sequence data. We generated a new sequence data from $P$. syringae pv. coryli NCPPB 4273, the reference pathotypestrain of the pathovar. The Illumina sequencing provided in total nearly 10 million 100-nt reads from the genomic DNA of the strain that passed the quality check. The genome size (nucleotides) of the strain was within the range of the previously sequenced and published $P$. syringae draft genomes (i.e., 6,096,328). The main genomic features of the draft genome are the following: number of reads, 6,398,721; number of contigs, 75; average contig size, 81,900; N50 (nt), 132,772; G+C content (\%), 59.2; and genome coverage, 155 . The sequence of the assembly was deposited in NCBI GenBank under the following accession number: $P$. syringae pv. coryli NCPPB 4273=AWQP00000000.

ANI and TETRA analyses. The newly sequenced $P$. syringae pv. coryli NCPPB 4273 genome, together with those of 33 phytopathogenic $P$. syringae pathovars and related Pseudomonas spp. strains, representative of seven of the nine genomospecies described by Gardan et al. (16) after their DDH and ribotyping assessment were cross compared to reveal their total similarities in terms of the amount of sequence identity. Two Pseudomonas spp., namely $P$. fluorescens A506 and $P$. putida UW4, were used as outgroups. The ANI value comparisons and calculations are shown in Figure 1 and Supplemental Table 1, and the corresponding TETRA

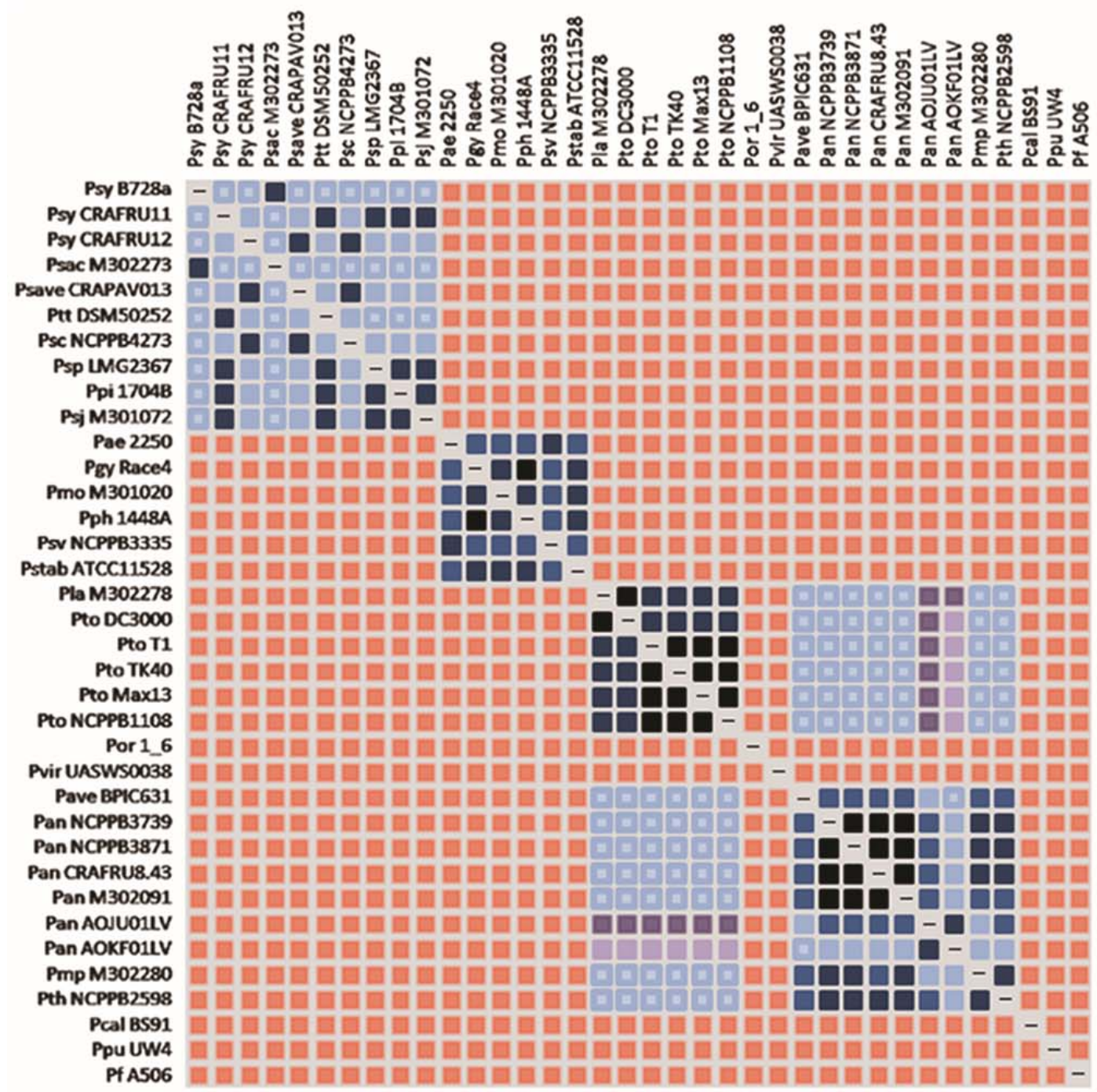
90919293949596979899100

$\leq 90$

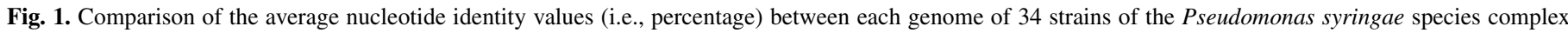

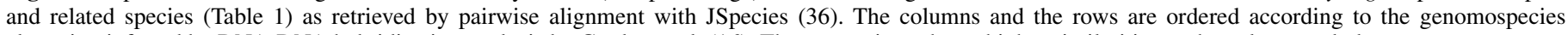
clustering inferred by DNA-DNA hybridization analysis by Gardan et al. (16). The comparison shows higher similarities as the colors get darker. 
values are shown in Supplemental Table 2. The ANI and TETRA analyses, in many cases, revealed a consistent demarcation among the strains of the $P$. syringae pathovars and related species, thereby allowing the circumscription of putative species boundaries. In fact, the strains of genomospecies 2 sensu Gardan et al. (16), namely $P$. syringae pathovars aesculi (2250), glycinea (race 4), mori (MAFF 301020), phaseolicola (1448A), tabaci (ATCC 11528 ), and P. savastanoi pv. savastanoi (NCPPB 3335) had ANI values between 97.74 and $99.45 \%$ in any reciprocal comparison and lower than $90 \%$ compared with the other P. syringae and outgroup strains. P. syringae pv. oryzae 1_6 (genomospecies 4), $P$. viridiflava UASWS0038 (genomospecies 6), and P. cannabina pv. alisalensis BS91 (genomospecies 9) had ANI values lower than $89 \%$ when compared with all other Pseudomonas strains.

The strains included in genomospecies 1 by the DDH and ribotyping analyses of Gardan et al. (16) and other studies $(34,46)$ and belonging to the $P$. syringae pv. syringae sensu strictu (16) had ANI values lower than $90 \%$ when compared with the strains of the other genomospecies and a genomic similarity ranging between 96 and $99 \%$ for intra-genomospecies comparison. However, it should be noted that the $P$. syringae pathovars syringae (B728a) and aceris (MAFF 302273) strains had ANI values between 95 and $96 \%$ when compared with the other strains of genomospecies 1 , revealing a lower genomic similarity that should be further investigated. The strains belonging to genomospecies 3 ( $P$. syringae $\mathrm{pv}$. lachrymans $\mathrm{MAFF} 302278, P$. syringae $\mathrm{pv}$. tomato DC3000, T 1, TK40, Max13, and NCPPB 1108) and genomospecies 8 (P. avellanae BPIC 631, P. syringae pv. theae NCPPB 2598, $P$. syringae pv. actinidiae NCPPB 3739, NCPPB 3871, CRAFRU 8.43, LV-11, and LV-17) had very high ANI values in the intra-genomospecies reciprocal comparison (Fig. 1). In addition, $P$. syringae pv. morsprunorum MAFF 302280 had ANI values higher than $96.3 \%$ when compared with the strains of genomospecies 8; therefore, it should be included in this genomospecies. However, the strains of genomospecies 3 and 8 , in many cases, had ANI values between 95 and $96 \%$ when they were reciprocally compared, thus revealing a very close taxonomic and phylogenetic relationship between these two genomospecies. The TETRA analysis always confirmed these results, with values for the genomospecies demarcation always higher than 0.99 in any reciprocal comparison. This analysis was intended to verify and confirm whether an alignment-free genomic feature can be used to circumscribe bacterial species (41). P. fluorescens A506 and $P$. putida UW4, used as outgroups, always had ANI values lower than $86 \%$ in any reciprocal comparison with the phytopathogenic pseudomonads.

MLSA and consensus split network analyses. A phylogenetic tree of the concatenated DNA sequences based on the ML method of the seven housekeeping genes, namely $d n a Q$, gltA, pheS, recA, $r p o B$, serS, and tyrS (a total of 3,003 nucleotides) in 34 phytopathogenic pseudomonads, representative of seven genomospecies sensu Gardan et al. (16) and P. fluorescens and P. putida used as outgroups, is shown in Figure 2. The robustness of the tree was strongly supported by the SH test (Table 2). The dendrogram consistently confirmed the distinctiveness of the seven genomospecies inferred by DDH and ribotyping by Gardan et al. (16) and the present ANI assessment. In addition, the tree confirmed the close relationships between the strains of genomospecies 3 and 8 inferred by the ANI and TETRA analyses. Similar to the ANI and TETRA analyses, MLSA also confirmed $P$. syringae

TABLE 1. List of Pseudomonas syringae pathovars and related species strain genomes used in this study for genomic and phylogenetic analyses ${ }^{\text {a }}$

\begin{tabular}{|c|c|c|c|c|c|}
\hline Strain & Strain code & Strain acronym & Accession number & Genomospecies & MLSA \\
\hline P. syringae pv. syringae $\mathrm{B} 728 \mathrm{a}$ & Psy B728a & Psy1 & NC_007005 & 1 & 1 \\
\hline P. syringae pv. syringae CRAFRU11 & Psy CRAFRU11 & Psy2 & ATSU00000000 & 1 & 1 \\
\hline P. syringae pv. syringae CRAFRU12 & Psy CRAFRU12 & Psy3 & ATSV00000000 & 1 & 1 \\
\hline P. syringae pv. avellanae CRAPAV013 & Psave CRAPAV013 & Psave & AKCJ00000000 & 1 & 1 \\
\hline P. syringae pv. aceris MAFF302273 PT & Psac M302273 & Psac & AEA000000000 & 1 & 1 \\
\hline P. syringae pv. aptata DSM50252 & Ptt DSM50252 & $\mathrm{Ptt}$ & AEAN00000000 & 1 & 1 \\
\hline P. syringae pv. coryli $\mathrm{NCPPB} 4273 \mathrm{PT}$ & Psc NCPPB4273 & Psc & AWQP00000000 & 1 & 1 \\
\hline P. syringae pv. panici LMG2367 PT & Psp LMG2367 & Psp & ALAC00000000 & 1 & 1 \\
\hline P. syringae pv. pisi $1704 \mathrm{~B}$ & Ppi 1704B & Ppi & AEAI00000000 & 1 & 1 \\
\hline P. syringae pv. japonica MAFF301072 PT & Psj M301072 & Psj & AEAH00000000 & 1 & 1 \\
\hline P. syringae pv. aesculi 2250 & Pae 2250 & $\mathrm{Pae}$ & ACXT00000000 & 2 & 2 \\
\hline$P$. syringae pv. glycinea race 4 & Pgy Race4 & Pgy & ADWY00000000 & 2 & 2 \\
\hline P. syringae pv. mori MAFF301020 & Pmo M301020 & Pmo & AEAG00000000 & 2 & 2 \\
\hline P. syringae pv. phaseolicola $1448 \mathrm{~A}$ & Pph 1448A & Pph & NC_005773, NC_007274, NC_007275 & 2 & 2 \\
\hline P. savastanoi pv. savastanoi NCPPB3335 & Psv NCPPB3335 & Psv & ADMI02000000 & 2 & 2 \\
\hline P. syringae pv. tabaci ATCC11528 & Pstab ATCC11528 & Pstab & ACHU00000000 & 2 & 2 \\
\hline P. syringae pv. lachrymans MAFF302278 PT & Pla M302278 & Pla & AEAM00000000 & / & 3 \\
\hline P. syringae pv. tomato DC 3000 & Pto DC3000 & Pto1 & NC_004578, NC_004632, NC_004633 & 3 & 3 \\
\hline P. syringae pv. tomato $\mathrm{T} 1$ & Pto T1 & Pto2 & ABSM 00000000 & 3 & 3 \\
\hline P. syringae pv. tomato TK40 & Pto TK40 & Pto3 & ADFY00000000 & 3 & 3 \\
\hline P. syringae pv. tomato Max 13 & Pto Max13 & Pto4 & ADFZ00000000 & 3 & 3 \\
\hline P. syringae pv. tomato NCPPB 1108 & Pto NCPPB 1108 & Pto5 & ADGA00000000 & 3 & 3 \\
\hline P. syringae pv. oryzae $1 \_6$ & Por 1_6 & Por & ABZR00000000 & 4 & 4 \\
\hline P. viridiflava UASWS 0038 & Pvir UASWS0038 & Pvir & AMQP00000000 & 6 & 6 \\
\hline P. avellanae BPIC631 T/PT & Pave BPIC631 & Pave & ATDK00000000 & 8 & 8 \\
\hline P. syringae pv. actinidiae NCPPB3739 PT & Pan NCPPB3739 & Pan1 & AFTH00000000 & 8 & 8 \\
\hline P. syringae pv. actinidiae NCPPB3871 & Pan NCPPB3871 & Pan2 & AFTF00000000 & 8 & 8 \\
\hline P. syringae pv. actinidiae CRAFRU8.43 & Pan CRAFRU8.43 & Pan3 & AFTG00000000 & 8 & 8 \\
\hline P. syringae pv. actinidiae MAFF302091 & Pan M302091 & Pan4 & AEAL00000000 & 8 & 8 \\
\hline$P$. syringae pv. actinidiae LV-11 & Pan AOJU01LV & Pan5 & AOJU00000000 & 8 & 8 \\
\hline P. syringae pv. actinidiae LV-17 & Pan AOKF01LV & Pan6 & AOKF00000000 & 8 & 8 \\
\hline P. syringae pv. morsprunorum M302280 PT & Pmp M302280 & Pmp & AEAE00000000 & 8 & 8 \\
\hline P. syringae pv. theae NCPPB2598 PT & Pth NCPPB2598 & Pth & ATDJ00000000 & 8 & 8 \\
\hline P. cannabina pv. alisalensis $\mathrm{BS} 91 \mathrm{PT}$ & Pcal BS91 & Pcal & ID 2516653056 & 9 & 9 \\
\hline P. putida UW4 & Ppu UW4 & Ppu & NC_019670 & Outgroup & \\
\hline P. fluorescens A506 & Pf A506 & Pf & NC_017911 & Outgroup & \\
\hline
\end{tabular}

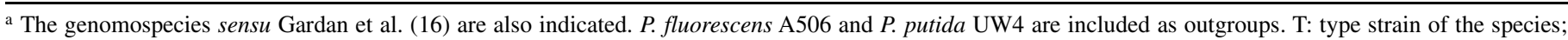

PT: pathotype strain of the pathovar. MLSA, multilocus sequence typing analysis. 
pv. lachrymans MAFF 302278 as a member of genomospecies 3 and $P$. syringae pv. morsprunorum MAFF 302280 as a member of genomospecies 8 . The representative strains of genomospecies 4 ( $P$. syringae pv. oryzae 1_6), 6 (P. viridiflava UASWS0038), and 9 (P. cannabina pv. alisalensis BS91) clustered separately from the other groups. Finally, the two outgroups P. fluorescens A506 and $P$. putida UW4 clustered far apart. The dendrogram built on with the Bayesian method provided the same strain genomes clustering as ML (Fig. 3). Both trees were supported by high bootstrap values. The consensus-split network, built for inferring unrooted phylogenetic networks and based on the concatenated alignment of the 3,003 nucleotides of the seven housekeeping genes, provided a significant and identical phylogenetic analysis compared with the ML tree and was supported by high bootstrap values (Figs. 4 and 5).

\section{DISCUSSION}

This study confirms the robustness of the combination of genomic and phylogenetic approaches in revealing taxonomic relationships among closely related bacterial strains for the definition of species $(42,51,52)$. We applied the ANI/TETRA analyses jointly with MLSA, using both ML and Bayesian methods, and split consensus network analyses for seven housekeeping genes in 34 strains belonging to the plant-pathogenic $P$. syringae complex and related species, and we confirmed the occurrence of putative species generally reflecting the classification of Gardan et al. (16), which was inferred through DDH and ribotyping analyses. Our analyses included several pathotype strains of the $P$. syringae pathovars and the type strain of $P$. avellanae, providing additional value to this assessment. The joint utilization of genomic data and phylogenetic analyses is very useful for providing new opportunities to infer bacterial species using a robust, reliable, and inexpensive approach $(42,51,52)$. In addition to the seminal studies proposing and confirming ANI analysis as a valuable method for the demarcation of bacterial species $(20,27,41)$, assessments of bacterial taxa using this approach that led to the redefinition of bacterial species have been reported for Acinetobacter spp. (10), Neisseria spp. (3), and P. avellanae (46). The present study con- firms that ANI/TETRA analyses combined with MLSA and consensus network can substitute for DDH to provide a reliable definition of bacterial species of the $P$. syringae complex.

Our study highlights the clear distinctiveness of the strains of genomospecies 1, 2, 4, 6, and 9. According to Gardan et al. (16), genomospecies 1 includes $P$. syringae pv. syringae strains sensu stricto and some other $P$. syringae pathovars, and their DDH values ranged between 71 and $90 \%$. The present study, taking into account strains other than those assessed by DDH and ribotyping, confirmed these conclusions. Within the group, however, there were some cases of strain comparison with ANI values between 95 and $96 \%$, such as $P$. syringae pv. aceris MAFF 302273 and $P$. syringae pv. syringae B728a. MLSA trees and split consensus network analyses confirmed the distinctiveness of genomospecies 1 from the other genomic clusters. $P$. syringae pv. syringae, as revealed by the DDH assessment, is a heterogeneous group of strains characterized by their unrestricted pathogenicity and genetic heterogeneity $(32,47,59)$, consequently, genomic variability should be expected. In contrast, the strains of genomospecies 2 used in this work formed tight and homogeneous clusters with ANI values consistently greater than $97.5 \%$, and $P$. syringae pv. oryzae 1_6, (genomospecies 4), P. viridiflava UASWS0038 (genomospecies 6), and $P$. cannabina pv. alisalensis BS91 (genomospecies 9) were well demarcated from all other genomospecies.

TABLE 2. Shimodaira-Hasegawa (SH) test for the congruence of phylogenetic trees inferred with maximum likelihood analysis and performed on 15 bifurcarting trees ${ }^{\mathrm{a}}$

\begin{tabular}{lc}
\hline Gene & SH \\
\hline dnaQ & 1.000 \\
gltA & 0.998 \\
pheS & 0.992 \\
recA & 0.999 \\
rpoB & 0.939 \\
serS & 1.000 \\
tyrS & 0.998 \\
Concatenated & 0.982 \\
\hline
\end{tabular}

a All values are significant at $\alpha=0.05$.

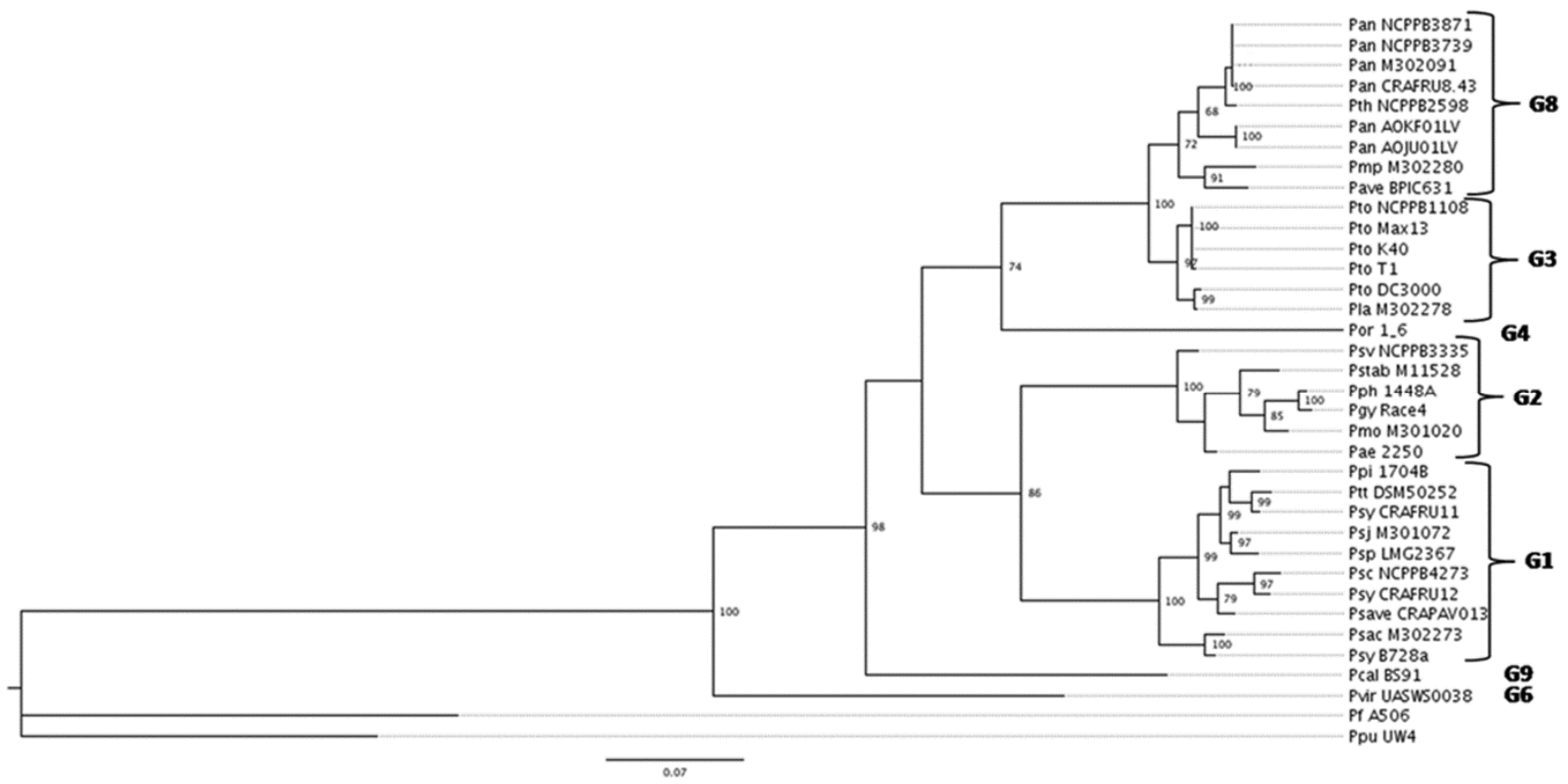

Fig. 2. Phylogenetic relationships among 34 strains of the Pseudomonas syringae species complex and related species as obtained with multilocus sequence typing analysis using 3,003 concatenated nucleotides of seven housekeeping genes (dnaQ, gltA, pheS, recA, rpoB, serS, and tyrS) with bootstrap values greater than $65 \%$ (1,000 replicates) shown at the nodes. The phylogenetic tree was built with the maximum likelihood (ML) method and with JC69 as the best substitution model using the PHYLIP package. P. fluorescens A506 (Pf) and P. putida UW4 (Ppu) were included as outgroups. G: genomospecies number sensu Gardan et al. (16). 
To establish the reliability and threshold accuracy of ANI analysis for robust species demarcation, a boundary of 95 to $96 \%$ was proposed $(27,41)$. The present study confirms that such a threshold reveals the occurrence of very closely related species that may represent cases of splitting populations or the convergence of different species to the same ecological niche. In fact, we found that some phytopathogenic Pseudomonas strains of genomospecies 3 and 8 had ANI values between 95 and $96 \%$. The

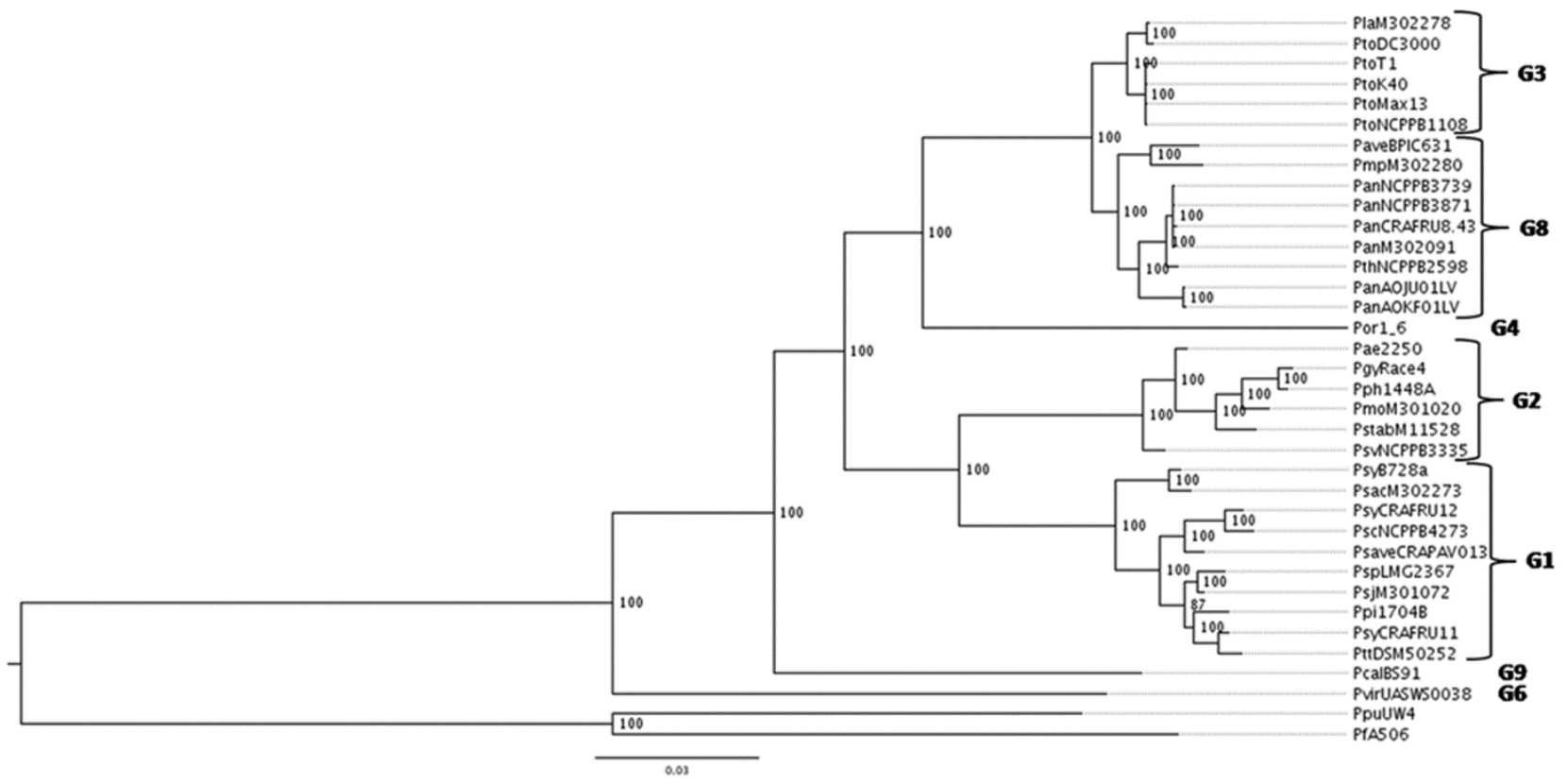

Fig. 3. Phylogenetic relationships among 34 strains of the Pseudomonas syringae species complex and related species as obtained with multilocus sequence typing analysis using 3,003 concatenated nucleotides of seven housekeeping genes (dnaQ, gltA, pheS, recA, rpoB, serS, and tyrS) inferred with the Bayesian method. Bootstrap values greater than $65 \%(1,000,000$ generations) are shown at the nodes. The interior node values of the tree are clade credibility values based on the posterior credibility values produced by MrBayes. G, genomospecies number sensu Gardan et al. (16).

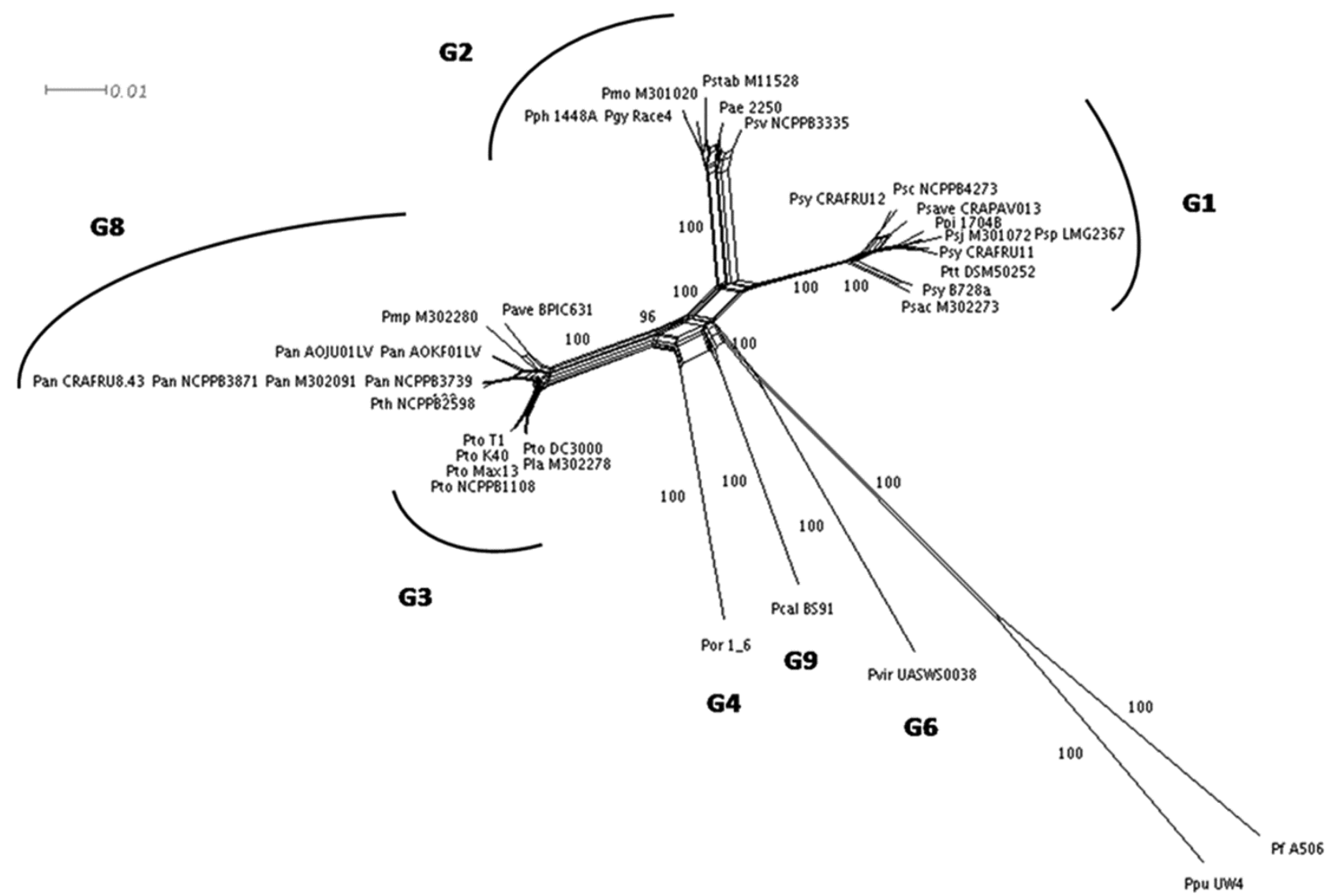

Fig. 4. Split consensus network of the concatenated 3,003 nucleotides of seven housekeeping genes (dnaQ, gltA, pheS, recA, rpoB, serS, and tyrS) for the 34 strains of the Pseudomonas syringae species complex and related species. Bootstrap values are shown at the main nodes. The split consensus network confirmed the results obtained with average nucleotide identity and multilocus sequence typing analysis analyses. G: genomospecies number sensu Gardan et al. (16). 
close relationships between genomospecies 3 and 8 had been revealed previously by ribotyping analysis (16) of three strains of genomospecies 3 which were included in genomospecies 8 , and several restriction fragments were observed to be common to all strains of both genomospecies. The close relationship between the genomospecies 3 and 8 was also demonstrated by hrp gene and gyrB and rpoD genes analysis $(24,44)$. Further studies are necessary to elucidate the evolutionary trajectories of these two groups of phytopathogens that infect both woody and herbaceous host plant species, leading to their current taxonomic similarity. A case of convergent evolution to the same host, namely Corylus avellana, was found for Pseudomonas strains of genomospecies 1 (i.e., $P$. syringae pv. avellanae and $P$. syringae pv. coryli) and $8(P$. avellanae) $(46,56)$. We also confirmed that $P$. syringae pv. morsprunorum MAFF 302280 is a member of genomospecies 8 (46). Notably, Gardan et al. (16) included the pathotype strain of $P$. syringae pv. morsprunorum $(\mathrm{CFBP} 2351=\mathrm{NCPPB} 2995=\mathrm{LMG}$ 5075t2=MAFF 302280) in their study and classified it as a member of genomospecies 3, even though the ribotyping analysis clearly showed its very close relationship to the strains of genomospecies 8 and with $P$. syringae pv. theae in particular. Young et al. (60) claimed that CFBP 2351 is unsuitable as a pathotype strain for this pathovar, and Gardan et al. (16) stated that it is not pathogenic. Consequently, Gardan et al. (16) and Bull et al. (5) proposed CFBP 2116, which was included in genomospecies 2, as a possible reference pathotype strain for the pathovar morsprunorum. Unfortunately, genomic data are not available for this strain. It should be added that, primarily based on biochemical tests (30), $P$. syringae pv. morsprunorum is, in turn, subdivided into two races, namely race 1 and race 2 . The strains of the two races appear quite homogenous and are thus reliably identified and distinguished according to biochemical and physiological tests (55) as well as by repetitive-sequence polymerase chain reaction (PCR) typing, MLST, and 16S rRNA gene sequence analyses $(18,25,31)$. According to these criteria, $P$. syringae pv. morsprunorum MAFF 302280 belongs to race 2, similar to other pathogenic strains $(18,31)$, whereas there are no race indications for CFBP 2116 even though it should, presumably, belong to race 1. Currently, genomic data for the strain comparisons of race 1 are not available, but the possibility that members of this race form a distinct species from the strains of race 2 (i.e., genomospecies 8) appears reasonable $(7,46)$. Additional analyses of other strains of both races 1 and 2 using a phylogenomic approach can contribute to the clarification of the existence of different Pseudomonas species that have pathogenically converged to the same host plants, namely Prunus avium, Prunus cerasus, and Prunus domestica. This study also confirms that the strains isolated from Actinidia deliciosa and A. chinensis in New Zealand, the so called "less virulent" $P$. syringae pv. actinidiae strains LV 11 and LV 17, should be included within the $P$. avellanae species (46). In fact, the close relationships between these strains and members of genomospecies 8 , particularly $P$. syringae pv. theae, have been also demonstrated by other MLSA and genome comparisons $(8,37)$.

The placement of $P$. syringae pv. lachrymans into the genomospecies scheme proposed by Gardan et al. (16) raises some questions. In their DDH and ribotyping analyses, these authors assessed two strains, CFBP 1644 and CFBP 2440, which were placed into genomospecies 2 and 3, respectively. Neither is the pathotype strain of the pathovar. The present study assessed the pathotype strain of the pathovar, CFBP 2104=NCPPB 1436=MAFF 302278, which was included within genomospecies 2. The pathovar lachrymans is heterogeneous in terms of both virulence and molecular features as inferred by PCR-restriction fragment length polymorphism of the internal transcribed spacer 1 (ITS1) region and amplification of DNA fragments surrounding rare restriction sites $(35,39)$. This pathovar is presumed to represent a case of pathogenic convergence of different species to the same host plants. Similarly, additional strain genome assessments using the approach of the present study could provide novel insights into the taxonomic and phylogenetic relationships of the P. syringae pvs. morsprunorum and lachrymans.

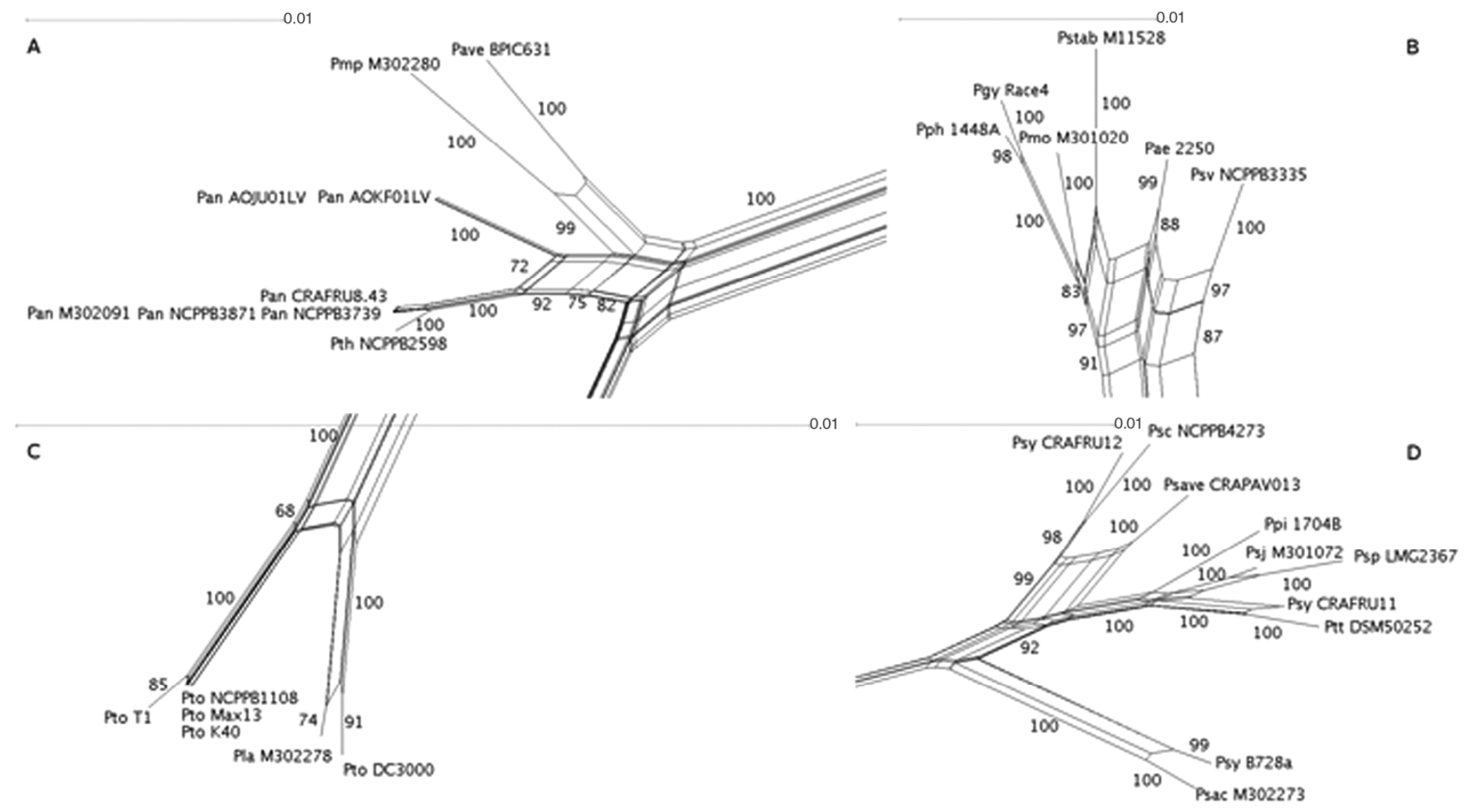

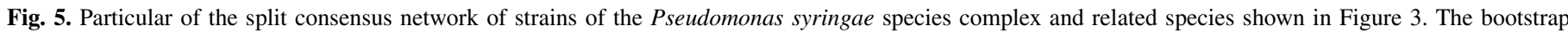
values are shown at each node. A, genomospecies 8; $\mathbf{B}$, genomospecies 2; $\mathbf{C}$, genomospecies 3; and $\mathbf{D}$, genomospecies 1 . Table 1 provides the strain codes. 
This study provides the basis for a further reliable species demarcation within the $P$. syringae species complex and contributes to the definition of more robust genomic and taxonomic relationships among the different pathovars in each genomospecies. Further analyses, possibly taking into account all the missing type and pathotype strains of the complex, would provide additional refinement of the current species demarcation (42). Another fundamental requirement for bacterial species definition and identification is their phenotypic demarcation (54). Similar to Gardan et al. (16), we are currently unable to provide a reliable phenotypic trait or technique to identify the genomospecies here inferred by the genomic and phylogenetic approaches. Modern approaches such as MALDI-TOF and high-field cyclotron Fourier transform mass spectrometry techniques can provide robust phenotypic assessments (42).

Concerning the composition of phytopathogenic bacterial species as revealed by the present study, a possible scenario might be the occurrence, within one single species, of some or many different pathovars, differentiated mainly by their accessory genome elements coding for virulence or environmental fitness traits and enabling them to colonize the host plant(s) and cause disease. Each single pathovar of a species may represent a distinct biological unit currently adapted to a well-defined niche(s), (i.e., the host plant(s) and the linked environment). Within this context, both the phylogenomic $(51,52)$ and ecological-evolutionary $(26$, 57) species concepts could be pertinent for explaining the relevant biological diversity and adaptive capability of bacterial phytopathogens, even though the diversity of each pathovar within a species should require the confirmation of its ecological distinctness through phenotypic characterization $(19,42)$. The possibility of performing extensive and standardized host range and phenotypic tests with many strains of different pathovars can possibly assist phytobacteriologists for better determining the boundaries of these ecological units.

\section{ACKNOWLEDGMENTS}

The study was financed by the Italian Ministry of Agriculture, Food and Forestry, project ARDICA: "Azioni di ricerca e difesa al cancro batterico dell'Actinidia (Psa)."

\section{LITERATURE CITED}

1. Almeida, N. F., Yan, S., Cai, R., Clarke, C. P., Morris, C. E., Schaad, N. W., Lacy, G. H., Jones, J.B., Castillo, J. A., Bull, C. T., Leman, S., Guttman, D. S., Setubal, J. C., and Vinatzer, B. A. 2010. PAMBD, a multilocus sequence typing and analysis database and website for plantassociated and plant-pathogenic microorganisms. Phytopathology 100: 208-215.

2. Altschul, S. F., Madden, T. L., Schäffer, A. A., Zhang, J., Zhang, Z., Miller, W., and Lipman, D. J. 1997. Gapped BLAST and PSI-BLAST: A new generation of protein database search programs. Nucleic Acids Res. 25:3389-3402.

3. Bennett, J. S., Jolley, K. A., Earle, S. G., Corton, C., Bentley, S. D., Parkhill, J., and Maiden, M. C. 2012. A genomic approach to bacterial taxonomy: An examination and proposed reclassification of species within the genus Neisseria. Microbiology 158:1570-1580.

4. Bull, C. T., Clarke, C. R., Cai, R., Vinatzer, B. A., Jardini, M. T., and Koike, S. T. 2011. Multilocus sequence typing of Pseudomonas syringae sensu latu confirms previously described genomospecies and permits rapid identification of $P$. syringae pv. coriandricola and $P$. syringae pv. apii causing bacterial leaf spot on parsley. Phytopathology 101:847-858.

5. Bull, C. T., De Boer, S. H., Denny, T. P., Firrao, G., Fischer-Le Saux, M., Saddler, G. S., Scortichini, M., Stead, D. E., and Takikawa, Y. 2010. Comprehensive list of names of plant pathogenic bacteria, 1980-2007. J. Plant Pathol. 92:551-592.

6. Bull, C. T., De Boer, S. H., Denny, T. P., Firrao, G., Fischer-Le Saux, M., Saddler, G. S., Scortichini, M., Stead, D. E., and Takikawa, Y. 2012. List of new names of plant pathogenic bacteria (2008-2010). J. Plant Pathol. 94:21-27.

7. Bultreys, A., and Kaluzna, M. 2010. Bacterial cankers caused by Pseudomonas syringae on stone fruit species with special emphasis on the patho- vars syringae and morsprunorum race 1 and 2. J. Plant Pathol. 91:S121S133.

8. Butler, M. I., Stockwell, P. A., Black, M. A., Day, R. C., Lamont, I. L., and Poulter, R. T. M. 2012. Pseudomonas syringae pv. actinidiae from recent outbreaks of kiwifruit bacterial canker belong to different clones that originated in China. PLoS One 8:e57464.

9. Cai, R., Yan, S., Liu, H., Leman, S., and Vinatzer, B. A. 2011. Reconstructing host range evolution of bacterial plant pathogens using Pseudomonas syringae pv. tomato and its close relatives as a model. Inf. Gen. Evol. 11:1738-1751.

10. Chan, J. Z.-M., Halachev, M. R., Loman, N. J., Constantinidou, C., and Pallen, M. J. 2012. Defining the bacterial species in the genomic era: Insights from the genus Acinetobacter. BMC Microbiol. 12:302.

11. Coenye, T., Gevers, D., Van de Peer, Y., Vandamme, P., and Swings, J. 2005. Towards a prokaryotic genomic taxonomy. FEMS Microbiol. Rev. 29:147-167.

12. Delcher, A. L., Bratke, K. A., Powers, E. C., and Salzberg, S. L. 2007. Identifying bacterial genes and endosymbiont DNA with Glimmer. Bioinformatics 23:673-679.

13. Doudoroff, M., and Palleroni, N. 1974. Gram-negative aerobic rods and cocci. Genus I. Pseudomonas. Pages 217-243 in: Bergey's Manual of Determinative Bacteriology. Williams and Wilkins, Baltimore.

14. Dye, D. W., Bradbury, J. F., Goto, M., Hayward, R.A., Lelliott, R. A., and Schroth, M. N. 1980. International standards for naming pathovars of phytopathogenic bacteria and a list of pathovar names with standard in nomenclature. Rev. Plant Pathol. 59:153-168.

15. Federhen, S. 2003. The taxonomy project. Chapter 4 in: The NCBI Handbook, National Library of Medicine (US), National Centre for Biotechnology Information, Bethesda, MD.

16. Gardan, L., Shafik, H., Belouin, S., Broch, R., Grimont, F., and Grimont, P. A. D. 1999. DNA relatedness among the pathovars of Pseudomonas syringae and description of Pseudomonas tremae sp. nov. and Pseudomonas cannabina sp. nov. (ex Sutic and Dowson, 1959). Int. J. Syst. Bacteriol. 49:469-478.

17. Gevers, D., Cohan, F. M., Lawrence, J. G., Spratt, B. G., Coenye, T., Feil, E. J., Stackebrandt, E., Van de Peer, Y., Vandamme, P., Thompson, E. L., and Swings, J. 2005. Re-evaluating prokaryotic species. Nat. Rev. Microbiol. 3:733-739.

18. Gilbert, V., Legros, F., Maraite, H., and Bultreys, A. 2009. Genetic analyses of Pseudomonas syringae isolated from Belgian fruit orchards reveal genetic variability and isolate-host relationships within the pathovar syringae, and help identify both races of the pathovar mosrprunorum. Eur. J. Plant Pathol. 124:199-218.

19. Godreuil, S., Cohan, F., Shah, H., andTibayrenc, M. 2005. Which species concept for pathogenic bacteria? An e-debate. Infect. Gen. Evol. 5:375-387.

20. Goris, J., Konstantinidis, K. T., Klappenbach, J. A., Coenye, T., Vandamme, P., and Tiedjie, J. M. 2007. DNA-DNA hybridization values and their relationships to whole-genome sequence similarities. Int. J. Syst. Evol. Microbiol. 57:81-91.

21. Guindon, S., and Gascuel, O. 2003. A simple, fast, and accurate algorithm to estimate phylogenies by maximum likelihood. Syst. Biol. 52:696-704.

22. Höfte, M., and De Vos, P. 2007. Plant pathogenic Pseudomonas species. Pages 507-533 in: Plant-Associated Bacteria. Springer, Dordrecht, the Netherlands.

23. Huson, D. H., and Bryant, D. 2006. Application of phylogenetic networks in evolutionary studies. Mol. Biol. Evol. 23:254-267.

24. Inoue, Y., and Takikawa, Y. 2006. The $h r p Z$ and $h r p A$ genes are variable, and useful for grouping Pseudomonas syringae bacteria. J. Gen. Plant Pathol. 72:26-33.

25. Kaluzna, M., Ferrante P., Sobiczewski, P., and Scortichini, M. 2010. Characterization and genetic diversity of Pseudomonas syringae from stone fruits and hazelnut using repetitive-PCR and MLST. J. Plant Pathol. 92:781-787.

26. Koeppel, A., Perry, E. B., Sikorski, J., Krizanc, D., Warner, A., Ward, D. M., Rooney, A. P., Brambilla, E., Connor, N., Ratcliff, R. M., Nevo, E., and Cohan, F. M. 2008. Identifying the fundamental units of bacterial diversity: A paradigm shift to incorporate ecology into bacterial systematics. Proc. Natl. Acad. Sci. USA 105:2504-2509.

27. Konstantinidis, K. T., and Tiedje, J. M. 2005. Genomic insights that advance the species definition for prokaryotes. Proc. Natl. Acad. Sci. USA 102:2567-2572.

28. Kurtz, S., Phylippi, A., Delcher, A. L., Smoot, M., Shumway, M., Antonescu, C., and Salzberg, S. L. 2004. Versatile and open software for comparing large genomes. Genome Biol. 5:R12.

29. Lapage, S. P., Sneath, P. H. A., Lessel, E. F., Skerman, V. B. D., Seeliger, H. P. R., and Clark, V. A. 1992. International code of nomenclature of bacteria: Bacteriological code, 1990 revision. American Society for Microbiology Press, Washington, DC

30. Latorre, B. A., and Jones, A. L. 1979. Pseudomonas morsprunorum, the cause of bacterial canker of sour cherry in Michigan, and its epiphytic 
association with Pseudomonas syringae. Phytopathology 69:335-339.

31. Lee, Y. S., Koh, H. S., Sohn, S. H., Koh, Y. J., and Jung, J. S. 2012. Genetic diversity among Pseudomonas syringae pv. morsprunorum isolates from Prunus mume in Korea and Japan by comparative sequence analysis of 16S rRNA gene. Plant Pathol. J. 28:295-298.

32. Legard, D. E., Aquadro, C. F., and Hunter, J. E. 1993. DNA sequence variation and phylogenetic relationships among strains of Pseudomonas syringae pv. syringae inferred from restriction site maps and restriction fragment length polymorphism. Appl. Environ. Microbiol. 59:4180-4188.

33. Lindeberg, M., Cunnac, S., and Collmer, A. 2012. Pseudomonas syringae type III effector repertoires: Last words in endless arguments. Trends Microbiol. 20:199-208.

34. Loreti, S., Gervasi, F., Gallelli, A., and Scortichini, M. 2008. Further molecular characterization of Pseudomonas syringae pv. coryli. J. Plant Pathol. 90:57-64.

35. Manceau, C., and Brin, C. 2003. Pathovars of Pseudomonas syringae are structured in genetic populations allowing the selection of specific markers for their detection in plant samples. Pages 503-512 in: Pseudomonas syringae Pathovars and Related Pathogens. Kluwer Academic Publishers, Dordrecht, the Netherlands.

36. Marcelletti, S., Ferrante, P., Petriccione, M., Firrao, G., and Scortichini, M. 2011. Pseudomonas syringae pv. actinidiae draft genomes comparison reveals strain-specific features involved in adaptation and virulence to Actinidia species. PLoS One 6:e27297.

37. McCann, H., Rikkerink, E. H. A., Bertels, F., Fiers, M., Ashley, L., ReesGeorge, J., Andersen, M. T., Gleave, A. P., Haubold, B., Wohlers, M. W., Guttman, D. S., Wang, P. W., Straub, C., Vanneste, J., Rainey, P. B., and Templeton, M. D. 2013. Genomic analysis of the kiwifruit pathogen Pseudomonas syringae pv. actinidiae provides insights into the origins of an emergent plant disease. Plos Pathogens 9:e1003503.

38. Moore, E. R. B., Tindall, B. J., Martins Dos Santos, V. A. P., Pieper, D. H., Ramos, J.-L., and Palleroni, N. J. 2006. Nonmedical Pseudomonas. Pages 21-28 in: The Prokaryotes, vol. 6, Proteobacteria: Gamma subclass. Springer, New York.

39. Olczak-Waltman, H., Masny, A., Bartosewski, G., Plucienniczak, A., and Niemirowicz-Szcztt, K. 2007. Genetic diversity of Pseudomonas syringae pv. lachrymans strains isolated from cucumber leaves collected in Poland. Plant Pathol. 56:373-382.

40. Paradis, E., Claude, J., and Strimmer, K. 2004. APE: Analysis of phylogenetic and evolution in R language. Bioinformatics 20:289-290.

41. Richter, M., and Rosselló-Móra, R. 2009. Shifting the genomic gold standard for the prokaryotic species definition. Proc. Natl. Acad. Sci. USA 106:19126-19131.

42. Rosselló-Móra, R. 2012. Towards a taxonomy of Bacteria and Archaea based on interactive and cumulative data repositories. Environ. Microbiol. 14:318-334.

43. Sarkar, S. F., and Guttman, D. S. 2004. Evolution of the core genome of Pseudomonas syringae, a highly clonal, endemic plant pathogen. Appl. Environ. Microbiol. 70:1999-2012.

44. Sawada, H., Suzuki, F., Matsuda, I., and Saitou, N. 1999. Phylogenetic analysis of Pseudomonas syringae pathovars suggests the horizontal gene transfer of $\operatorname{argK}$ and the evolutionary stability of $h r p$ gene cluster. J. Mol. Evol. 49:627-644.

45. Schleifer, K.-H. 2009. Classification of Bacteria and Archaea: Past, present and future. Syst. Appl. Microbiol. 32:533-542.

46. Scortichini, M., Marcelletti, S., Ferrante, P., and Firrao, G. 2013. A genomic redefinition of Pseudomonas avellanae species. PLoS One 8:e75794.

47. Scortichini, M., Marchesi, U., Dettori, M. T., and Rossi, M. P. 2003. Genetic diversity, presence of $s y r B$ gene, host preference and virulence of Pseudomonas syringae pv. syringae strains from woody and herbaceous host plants. Plant Pathol. 52:277-286.

48. Shimodaira, H., and Hasegawa, M. 1999. Multiple comparisons of loglikelihoods with applications to phylogenetic inference. Mol. Biol. Evol. 16:1114-1116.

49. Shimodaira, H., and Hasegawa, M. 2001. CONSEL: For assessing the confidence of phylogenetic tree selection. Bioinformatics 17:1246-1247.

50. Skerman, V. B. D., McGowan, V., and Sneath, P. H. A. 1980. Approved lists of bacterial species. Int. J. Syst. Bacteriol. 3:255-420.

51. Staley, J. T. 2006. The bacterial species dilemma and the genomicphylogenetic species concept. Phil. Trans. R. Soc. B 361:1899-1909.

52. Staley, J. T. 2009. The phylogenomic species concept for Bacteria and Archaea. ASM Microbe Magazine 4:362-365.

53. Stolp, H., Starr, M. P., and Baignet, N. L. 1965. Problems in speciation in phytopathogenic pseudomonads and xanthomonads. Annu. Rev. Phytopathol. 3:231-264.

54. Tindall, B. J., Rosselló-Móra, R., Busse, H.-J., Ludwig, W., and Kämpfer, P. 2010. Notes on the characterization of prokaryote strains for taxonomic purposes. Int. J. Syst. Evol. Microbiol. 60:249-266.

55. Vicente, J. G., Roberts, S. J., Russell, K., and Alves, J. P. 2004. Identification and discrimination of Pseudomonas syringae isolates from wild cherry in England. Eur. J. Plant Pathol. 110:337-351.

56. Wang, P. W., Morgan, R. L., Scortichini, M., and Guttman, D. 2007. Convergent evolution of phytopathogenic pseudomonads onto hazelnut. Microbiology 153:2067-2073

57. Ward, D. M., Cohan, F. M., Bhaya, D., Heidelberg, J. F., Kühl, M., and Grossman, A. 2008. Genomics, environmental genomics and the issue of microbial species. Heredity 100:207-219.

58. Wayne, L. G., Brenner, D. J., Colwell, R. R., Grimont, P. A. D., Kandler, O., Krichevsky, M. I., Moore, L. H., Moore, W. E. C., Murray, R. G. E., Stackebrandt, E., Starr, M. P., and Truper, H. G. 1987. International Committee on Systematic Bacteriology. Report of the ad hoc committee on reconciliation of approaches to bacterial systematics. Int. J. Syst. Bacteriol. 37:463-464.

59. Young, J. M. 2010. Taxonomy of Pseudomonas syringae. J. Plant Pathol. 92:S1.5-S1.14.

60. Young, J. M., Bradbury, J. F., Davis, R. E., Dickey, R. S., Ercolani, G. L., Hayward, A. C., and Vidaver, A. K. 1991. Nomenclatural revision of plant pathogenic bacteria and a list of names 1980-1988. Rev. Plant Pathol. 70:211-221. 